\title{
Phase I trial of convection-enhanced delivery of IL13-Pseudomonas toxin in children with diffuse intrinsic pontine glioma
}

\author{
John D. Heiss, MD, ${ }^{1}$ Aria Jamshidi, MD, ${ }^{2}$ Smit Shah, BA, ${ }^{3}$ Staci Martin, PhD, ${ }^{4}$ \\ Pamela L. Wolters, PhD, ${ }^{4}$ Davis P. Argersinger, BS, ${ }^{1}$ Katherine E. Warren, MD, ${ }^{5}$ and \\ Russell R. Lonser, MD ${ }^{6}$
}

${ }^{1}$ Surgical Neurology Branch, National Institute of Neurological Disorders and Stroke, and ${ }^{4}$ Center for Cancer Research and ${ }^{5}$ Pediatric Oncology Branch, National Cancer Institute, National Institutes of Health, Bethesda, Maryland; 'Department of Neurological Surgery, University of Miami Miller School of Medicine, Miami, Florida; ${ }^{3}$ Rutgers University Robert Wood Johnson Medical School, Piscataway, New Jersey; and ${ }^{6}$ Department of Neurological Surgery, Ohio State University Wexner Medical Center, Columbus, Ohio

\begin{abstract}
OBJECTIVE In this clinical trial report, the authors analyze safety and infusion distribution of IL13-Pseudomonas exotoxin, an antitumor chimeric molecule, administered via intratumoral convection enhanced delivery (CED) in pediatric patients with diffuse intrinsic pontine glioma (DIPG).

METHODS This was a Phase I single-institution, open-label, dose-escalation, safety and tolerability study of IL13PE38QQR infused via single-catheter CED into 5 pediatric DIPG patients. IL13-PE38QQR was administered to regions of tumor selected by radiographic findings. Two escalating dose levels were evaluated: $0.125 \mu \mathrm{g} / \mathrm{mL}$ in cohort 1 and 0.25 $\mu \mathrm{g} / \mathrm{mL}$ in cohort 2. Real-time MRI was performed during intratumoral infusions, and MRI and MR spectroscopy were performed before and after the infusions. Clinical evaluations, including parent-reported quality of life (QOL), were assessed at baseline and 4 weeks post-infusion.

RESULTS Direct infusion of brainstem tumor with IL13-PE using the CED technique in patients with DIPG produced temporary arrest of disease progression in 2 of 5 patients, both of whom subsequently received a second infusion. All 5 patients showed signs of disease progression by 12 weeks after initial infusion. Two patients experienced transient cranial nerve deficits and lethargy after infusion, and these deficits resolved with corticosteroid treatment in both cases. No patient had radiographic evidence of acute or long-term treatment toxicity. Parent-reported QOL was consistent with medical outcomes.
\end{abstract}

CONCLUSIONS Even though IL13-PE delivered by CED did not reach the entire MRI-defined tumor volume in any patient, short-term radiographic antitumor effects were observed in 2 of the 5 patients treated. The patients' performance status did not improve. Drug delivery using multiple catheters may produce improved outcomes.

Clinical trial registration no:: NCT00088061 (clinicaltrials.gov)

https://thejns.org/doi/abs/10.3171/2018.9.PEDS17225

KEYWORDS DIPG; CED; convection-enhanced delivery; IL13-PE; Gd-DTPA; pediatric brain tumors; MR-spectroscopy; oncology

$\mathrm{D}$ IFFUSE intrinsic pontine glioma (DIPG) accounts for $75 \%-80 \%$ of childhood brainstem tumors. ${ }^{2}$ It typically arises in the pons, affects 3 - to 10 -yearold children, infiltrates and diffusely expands the pons, and causes progressive symptoms prompting medical attention within days to weeks. Patients may present with bilateral cranial nerve deficits, long tract signs, and gait difficulties. Characteristic MRI features include diffuse brainstem enlargement, hypointense or isointense signal on T1-weighted images, and hyperintense signal on T2weighted images. ${ }^{1}$ DIPG may not enhance at diagnosis, and enhancement patterns vary. Reduced H2K27me3 ex-

ABBREVIATIONS CED = convection-enhanced delivery; DIPG = diffuse intrinsic pontine glioma; IL-13 = interleukin 13; IL13R = IL-13 receptor; IPI = Impact of Pediatric Illness; KPS = Karnofsky Performance Status; LPS = Lansky Performance Status; MRS = MR spectroscopy; NAA = n-acetyl aspartate; $Q O L=$ quality of life; Vd = volume of distribution; $\mathrm{Vi}=$ volume of infusion.

SUBMITTED April 28, 2017. ACCEPTED September 12, 2018.

INCLUDE WHEN CITING Published online December 7, 2018; DOI: 10.3171/2018.9.PEDS17225. 
pression increases hypomethylation and promotes tumorigenesis. ${ }^{26}$ DIPG may have ACVR1 mutation and amplification of G1 checkpoint regulators. ${ }^{2,26}$

A 54- to 59-Gy radiotherapy dose is the only currently available treatment that improves clinical status and reduces DIPG size, ${ }^{2}$ but these effects are temporary and tumors progress within 3-6 months. ${ }^{27}$ The central brainstem location and infiltrative nature of DIPG precludes safe resection. ${ }^{31}$ Tumor resistance and poor blood-brain barrier drug permeability frustrate chemotherapy for DIPG. ${ }^{19}$ The median survival for children with DIPG is less than 1 year, and the 5-year survival rate is below 5\%. DIPG causes most pediatric brain tumor-related deaths. ${ }^{5}$

Agents with better drug delivery to the tumor, greater antitumor activity, and less toxicity may improve DIPG treatment outcomes. ${ }^{8}$ The clinical trial described used convection-enhanced delivery (CED) to distribute the investigational agent intratumorally into DIPGs. In small and large animal models, CED delivered agents safely and homogeneously into DIPG-size volumes without systemic toxicity. ${ }^{4}$

IL13-PE38QQR is a recombinant cytotoxic chimera of human interleukin 13 (IL-13) and the enzymatically active portion of Pseudomonas exotoxin A that effectively targeted glioma cell lines in preclinical studies. Most glioma cell lines and malignant glioma explants overexpress tumor-specific IL-13 (interleukin-4 [IL-4]-independent) receptor (IL13R), making it a viable therapeutic target. ${ }^{4-6}$ Tumor cells expressing the IL-13 receptor when exposed to IL13-PE38QQR bind the IL-13 component and internalize the Pseudomonas exotoxin component, which prevents adenosine diphosphate (ADP) ribosylation of elongation factor 2 , inhibits protein synthesis, and leads to apoptotic cell death. ${ }^{15}$

Preclinical and early clinical studies of IL13-Pseudomonas exotoxin demonstrated antitumor activity and a favorable risk-benefit profile for high-grade gliomas. ${ }^{13,18}$ However, IL13-PE had survival benefit similar to polifeprosan-carmustine wafers (Gliadel, Arbor Pharmaceuticals, LLC) in a Phase III randomized trial for recurrent glioblastoma. ${ }^{20}$ In this Phase I clinical trial report, we discuss the safety of IL13-PE38QQR administered intratumorally via CED in children with DIPG.

\section{Methods \\ Design}

This was a Phase I single-institution, open-label, doseescalation, safety and tolerability, pediatric study of IL13PE38QQR infused into DIPG via CED. A surrogate marker of IL13-PE38QQR distribution, Gd-DTPA, was co-infused. The initial $0.125 \mu \mathrm{g} / \mathrm{mL}$ concentration of IL13PE38QQR was well below the maximum intraparenchymal concentration of $0.5 \mu \mathrm{g} / \mathrm{mL}$ tolerated in supratentorial recurrent malignant glioma and 2 orders of magnitude above the $\mathrm{IC}_{50}$ of most glioma cell lines. ${ }^{6,21}$ Escalating dose levels were evaluated: $0.125 \mu \mathrm{g} / \mathrm{mL}$ in cohort 1 and 0.25 $\mu \mathrm{g} / \mathrm{mL}$ in cohort 2 . Vehicle control was not used because, unlike IL13-PE infusion, it did not offer the prospect of direct benefit to participants. ${ }^{25}$

The study was conducted under NIH protocol $09-\mathrm{N}$ -
0117, registered at clinicaltrials.gov (NCT00880061), and approved by the Combined Neurosciences Institutional Review Board. Informed consent was obtained from both legal guardians and assent from patients.

\section{Study Population and Treatment}

All enrolled patients satisfied inclusion criteria, including: 1) age $<18$ years, 2) clinical and radiological evidence of progressive DIPG, 3) $>2$ weeks after their last chemotherapy dose or neurosurgical procedure, and 4) > 4 weeks from last dose of radiation. With respect to this last criterion, the time since the last dose of radiation was $27,24,37,74$, and 42 weeks, respectively, for patients 1,2 , 3,4 , and 5. Baseline physical and neurological examinations detected neurological deficits and assessed capacity to undergo the planned surgical procedure. Five consecutive pediatric patients with progressive DIPG, defined as interval increase in tumor size on MRI and/or interval development of additional neurological signs of brainstem involvement, after prior standard DIPG radiotherapy were enrolled. Two additional patients failed screening. The enrolled patients ( 2 girls, 3 boys; mean age at initial infusion $13 \pm 5$ years, range 5-17 years) underwent 7 intratumoral infusions. Patients 1, 2, and 3 received the $0.125 \mu \mathrm{g} / \mathrm{mL}$ study drug concentration, and patient 3 received a second infusion of the same dose 4 weeks later. Patients 4 and 5 received the $0.25 \mu \mathrm{g} / \mathrm{mL}$ drug concentration, with patient 5 receiving a second infusion of that dose 4 weeks later.

\section{Clinical Safety and Outcome Measures}

Clinical functioning was evaluated at baseline (within 72 hours of enrollment) and at 4-week intervals after infusion, including 1) disease signs and symptoms, 2) corticosteroid dose, 3) Lansky Performance Status (LPS) or Karnofsky Performance Status (KPS) scores, and 4) health-related quality of life (QOL), using the 43-item Impact of Pediatric Illness (IPI) Parent Report Form. ${ }^{32,33}$ The IPI questionnaire uses a 5-point Likert-type scale (1 $=$ "not at all," $5=$ "a lot") to score 4 subscales (adaptive behavior, emotional functioning, medical/physical status, and cognitive problems) and provide a total score on a 0-100 scale. Higher scores indicate better QOL. Adverse and serious adverse events were recorded after infusion.

\section{Anatomical MRI and MR Spectroscopy}

Patients underwent preoperative MRI including: T1 (with and without contrast), T2, FLAIR, short- and longecho spectroscopy, and sometimes perfusion studies. Research MRI and MR spectroscopy (MRS) were repeated 1 week, 4 weeks, and then every 4 weeks after surgery. A neuroradiologist recorded tumor size, edema, hemorrhage, and contrast enhancement. Tumor margins were outlined on T2-weighted images, and tumor volume was calculated using Cavalieri's estimator. ${ }^{7}$ A neuroradiologist compared pre-infusion to post-infusion MRI and recorded change in tumor size, edema, hemorrhage, contrast enhancement, and intratumoral spectroscopic pattern.

\section{Preparation of IL13-PE38QQR and Gd-DTPA Infusate}

IL13-PE38QQR solution (InSys Therapeutics, Inc.) was 
thawed and diluted with $0.9 \%$ normal saline containing $0.2 \%$ human serum albumin; then Gd-DTPA solution (469 $\mathrm{mg} / \mathrm{mL}$ ) (Magnevist, Bayer Healthcare Pharmaceuticals, Inc.) was added to achieve a final Gd-DTPA concentration of $1 \mathrm{mM}$ (patient 1 only) or $5 \mathrm{mM}$ (patients 2-5) and a $0.125 \mu \mathrm{g} / \mathrm{mL}$ concentration of IL13-PE in patients $1-3(\mathrm{n}=$ $3)$ and $0.25 \mu \mathrm{g} / \mathrm{mL}$ in patients 4 and $5(\mathrm{n}=2)$. Trial enrollment concluded after the fifth patient because the manufacturer could not provide additional IL13-PE38QQR.

\section{Anesthesia and Monitoring}

General anesthesia and vital sign monitoring continued from initial intraoperative MRI scan until incision closure. Intravenous dexamethasone or methylprednisolone was given intra- and perioperatively.

\section{Frameless Stereotactic Surgical Approach}

Patients 1 and 2 underwent volumetric MRI scans the day before surgery that were uploaded to the surgical navigation system (StealthStation, Medtronic, Inc.). The navigation system registered surgical head position to the MRI. A right coronal burr hole was placed and the dura was opened. The Navigus catheter guide (Medtronic, Inc.) was aimed on a trajectory passing from the right coronal burr hole to a central DIPG target without breaching ventricles or blood vessels. A cannula (for patient 1, Vygon; for patient 2, NexGen Medical Systems, Inc.) was inserted through the catheter guide until its tip rested centrally in the DIPG. Intraoperative MRI (Achieva 1.5-T, Philips) confirmed appropriate cannula position before the cannula was connected to investigational agent in primed infusion tubing and a syringe. The syringe was loaded into an infusion pump (Medfusion 3500, Smiths Medical).

\section{Intraoperative MRI-Guided Stereotactic Approach}

The ClearPoint navigation system and SmartFlow cannula (MRI Interventions, Inc.) were used in patients 3, 4, and 5. General anesthesia was induced, the patient's head was fixed in an MRI-compatible head clamp (Integra LifeSciences Corp.), and surface coils were placed. An adherent localizing grid (MRI Interventions, Inc.) was placed on the right frontal scalp, an MRI scan obtained, and a skull entry point and surgical trajectory were devised that avoided ventricles, subarachnoid space, and blood vessels and placed the catheter tip centrally in the DIPG. The scalp was incised, right coronal burr hole placed, and dura opened. The ClearPoint cannula guide was adjusted using intraoperative MRI to direct the cannula into the tumor target. The SmartFlow cannula was inserted to the target, which was confirmed by MRI before infusion was initiated. The cannula was connected through primed tubing to a syringe in an infusion pump (Medfusion 3500, Medex, Inc.).

\section{Infusate Delivery}

Convective co-infusion of Gd-DTPA and IL13-PE started at $0.5 \mu \mathrm{L} /$ minute and increased at $0.5 \mu \mathrm{L} /$ minute increments every 10 minutes until reaching a maximum rate of $5 \mu \mathrm{L} /$ minute in patients 1 and 2 , or $10 \mu \mathrm{L} /$ minute in patients 3,4 , and 5 . Infusion progress was monitored by serial T1-weighted intraoperative MRI until infusion completion. The infusion rate was reduced if significant backflow along the catheter was observed. The infusion continued until the study agent (GD-DTPA signal) was distributed throughout the entire tumor, but no longer than 13 hours. The total volume of infusion (Vi) was recorded at infusion completion. The cannula was removed outside the MRI machine, and the incision was closed with sutures.

\section{Optional Second Infusion}

A second infusion was offered to those who tolerated the procedure well (no grade 3 dose-limiting toxicity related to the study agent or CED) and had improvement in neurological symptoms or performance score (KPS if $\geq$ 16 years; LPS if < 16 years) or stable or decreased steroid doses. The second infusion followed at least 4 weeks after the first, using the same method.

\section{Post-Infusion Clinical Monitoring}

After the procedure, the patients stayed overnight in the ICU before being transferred to an inpatient pediatric nursing unit, where they remained until discharge.

\section{Distribution of Infusate to Tumor Tissue}

The volume of distribution (Vd) was calculated using a connected thresholding technique for growing a region of interest on T1-weighted MR images (OsiriX, Pixmeo). ${ }^{32}$ A seed voxel was placed in the maximum intensity area around the catheter tip and grown using a specified threshold to include the entire infused $\mathrm{Vd}$.

\section{Statistical Analysis}

An unpaired Student t-test was used for comparisons, and $p$ values $<0.05$ were considered statistically significant.

\section{Results}

\section{Distribution of Infusate to Tumor Tissue}

The Gd-DTPA surrogate tracer $(1 \mathrm{mM}$ and $5 \mathrm{mM})$ was clearly identified within the perfused region on T1-weighted MRI during infusion. The 1-mM Gd-DTPA concentration resulted in a lower $\mathrm{Vd} / \mathrm{Vi}$ ratio (1.6) than $5-\mathrm{mM}$ Gd-DTPA $(2.8 \pm 1.1 ; \mathrm{p}=0.04,95 \%$ CI $0.046-2.351)$. The volumes infused and durations were, respectively, $2.8 \mathrm{~mL}$ and 10.1 hours (patient 1), $2.3 \mathrm{~mL}$ and 8.5 hours (patient 2), $2.7 \mathrm{~mL}$ and 7.3 hours (patient 3 , first infusion), $2.9 \mathrm{~mL}$ and 6.5 hours (patient 3 , second infusion), $1.1 \mathrm{~mL}$ and 3.3 hours (patient 4), $2.7 \mathrm{~mL}$ and 6.3 hours (patient 5, first infusion), and $2.8 \mathrm{~mL}$ and 6.2 hours (patient 5 , second infusion).

Infusion contrast first appeared around the cannula tip on MRI. The cannula tip position was adjusted to improve infusate delivery to tumor tissue. For example, after 100 minutes of patient 5's first infusion, MRI detected infusate pooling around the catheter tip, prompting retraction of the infusion catheter $18 \mathrm{~mm}$ in 3- to 5-mm increments until contrast-containing infusion solution entered surrounding tumor by CED. Real-time MRI documented increas- 


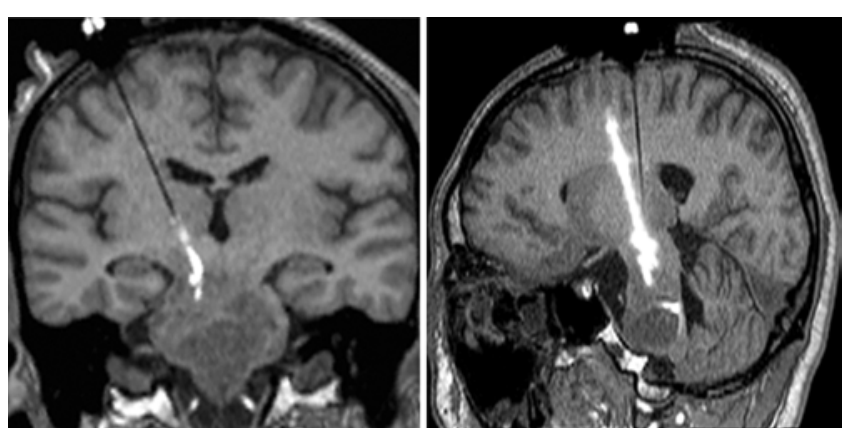

FIG. 1. Second infusion in patient 5. Intraoperative coronal (A) and sagittal-oblique (B) T1-weighted MR images demonstrating retrograde flow of infusate back into the old cannula tract from the infusion 4 weeks prior.

ing Vd thereafter. Patient 5 underwent a second infusion 4 weeks later, and after 125 minutes of infusion MRI detected retrograde filling in an old catheter tract (Fig. 1A and $\mathrm{B}$ ). Inserting the infusion catheter $10 \mathrm{~mm}$ deeper into the tumor stopped infusate reflux.

\section{Adverse Events}

Five serious adverse events occurred: elevated serum creatine kinase $(n=2)$, renal calculi $(n=1)$, somnolence $(\mathrm{n}=1)$, and suspected aspiration prompting hospitalization $(n=1)$. All serious adverse events resolved. There were 54 nonserious adverse events. Increasing weakness, cranial nerve dysfunction, dysarthria, and dysphagia occurred in the week after the procedure. Fifteen events remained unresolved 4 weeks after treatment (Table 1). No adverse event was a dose-limiting toxicity, defined as any grade 3 or greater toxicity probably or definitely-related to study drug. All patients died from DIPG, surviving 4.7-35.6 weeks after their first (or only) infusion of IL13-PE and 36.3-88.9 weeks after initial DIPG diagnosis (Table 2).

\section{Outcome Measures}

\section{Patient 1}

Hospital Course. A $10-\mathrm{cm}^{2}$ area of erythema was detected on the left buttock the day after infusion. Associated rhabdomyolysis and elevated creatine kinase resolved with IV hydration. The patient developed decreased responsiveness, increased drooling, and unequal and poorly reactive pupils after discontinuing methylprednisolone the following day. Repeat head CT was stable. Symptoms re- solved after a 4-mg dose of dexamethasone. A new right cranial nerve $(\mathrm{CN})$ VI palsy, left CN VII palsy, left-sided weakness, and worsening dysarthria resolved by discharge 8 days after surgery with continuing corticosteroid therapy.

Quality of Life. Scores on all 4 subscales decreased between baseline and 4-week post-infusion assessments, indicating reduced QOL post-infusion. The patient's score on the Adaptive Behavior subscale dropped from 72.7 at baseline to 56.84 weeks later based on self-care skill and motor function declines. The score on the Psychological Functioning subscale dropped from 70.5 to 56.8 because of less enjoyment of activities and more irritability. The score on the Medical/Physical Status subscale dropped from 75 to 61.5 due to daytime fatigue and increased pain. The score on the CNS Problems subscale decreased from 91.7 to 83.3 (Table 3 ) because of increased need for special educational services.

Anatomical MRI and MRS. Tumor volume and enhancement were unchanged on the 1-week post-infusion MRI. The left dorsolateral pons contained a microhemorrhage at the infusion catheter placement site. The tumor volume remained stable 4 weeks post-infusion, but the enhancing component enlarged slightly and had somewhat increased perfusion. At 8 weeks post-infusion, the tumor volume was still stable, but its enhancing component continued to enlarge and increase in perfusion. The 12 -week postoperative MRI demonstrated subtle increase in tumor size, increased central enhancement, and fourth ventricle impingement. The blood volume increased within the enhancing mass on perfusion imaging. The patient withdrew from the study, enrolled in another study, and died 12 weeks later.

Pre-infusion MRS showed reduced concentration of nacetyl aspartate (NAA). MRS performed 2 days, 8 weeks, and 12 weeks post-infusion demonstrated marked elevation of choline and reduction of NAA.

\section{Patient 2}

Hospital Course. The day after surgery, the patient had a left CN V palsy, worsening right-sided weakness, and new left-sided weakness. These deficits resolved by discharge 6 days after infusion.

Quality of Life. The patient did not have complete QOL data for either time point.

Anatomical MRI and MRS. The 1-week postoperative MRI demonstrated stable lesion size and new hemosid-

TABLE 1. Cumulative adverse events: severity and causality

\begin{tabular}{|c|c|c|c|c|c|c|}
\hline $\begin{array}{l}\text { Maximum } \\
\text { Severity }\end{array}$ & $\begin{array}{l}\text { Any Relationship } \\
\qquad(n=54)\end{array}$ & $\begin{array}{l}\text { Attribution to Study Procedure } \\
\qquad(\mathrm{n}=21)\end{array}$ & $\begin{array}{l}\text { Attribution to Study Drug } \\
\qquad(n=16)\end{array}$ & $\begin{array}{l}\text { Unrelated to Study } \\
\qquad(\mathrm{n}=17)\end{array}$ & $\begin{array}{l}\text { Resolved } \\
(n=39)\end{array}$ & $\begin{array}{l}\text { Unresolvec } \\
\qquad(n=15)\end{array}$ \\
\hline Grade 1 & $29(54 \%)$ & 13 & 8 & 8 & 27 & 2 \\
\hline Grade 2 & $23(43 \%)$ & 8 & 8 & 7 & 11 & 12 \\
\hline Grade 3 & $2(3 \%)$ & 0 & 0 & 2 & 1 & 1 \\
\hline Grade 4 & 0 & 0 & 0 & 0 & 0 & 0 \\
\hline Grade $5^{*}$ & 0 & 0 & 0 & 0 & 0 & 0 \\
\hline
\end{tabular}

* All patients ultimately died from progression of the underlying disease (documented MRI progression of disease process beyond its extent at the time of infusion). 
TABLE 2. Treatments and their timing before IL13-PE infusion

\begin{tabular}{cccccll}
\hline $\begin{array}{c}\text { Pt } \\
\text { No. }\end{array}$ & $\begin{array}{c}\text { Dx to End } \\
\text { of RT (wks) }\end{array}$ & $\begin{array}{c}\text { End of RT to End } \\
\text { of Chemo (wks) }\end{array}$ & $\begin{array}{c}\text { End of Chemo to } \\
\text { IL13-PE Infusion (wks) }\end{array}$ & $\begin{array}{c}\text { 1st IL13-PE Infusion } \\
\text { to Death (wks) }\end{array}$ & $\begin{array}{c}\text { Dx to Death } \\
\text { (wks) }\end{array}$ & Chemo Regimens \\
\hline 1 & 7.9 & 18.6 & 8.0 & 22.0 & 56.4 & Vandetanib \\
\hline 2 & 8.0 & 0.9 & 22.9 & 4.7 & 36.3 & Vorinostat \\
\hline 3 & 8.0 & 32.9 & 4.1 & $35.6 \dagger$ & 80.9 & a) Cetuximab \& irinotecan; b) MK2206 \\
\hline 4 & 5.4 & 69.1 & 5.0 & 9.3 & 88.9 & a) PEG irinotecan; b) VOIT; c) avastin \& etoposide \\
\hline 5 & 6.3 & 38.3 & 4.0 & $24.3 \dagger$ & 72.9 & a) Temozolomide, vorinostat, avastin; b) temsirolimus \\
\hline
\end{tabular}

Chemo = chemotherapy; Dx = diagnosis; PEG = polyethylene glycol; $\mathrm{pt}=$ patient; $\mathrm{RT}$ = radiation therapy; VOIT = vincristine, oral irinotecan, and temozolomide.

$\dagger$ Patients received second IL13-PE infusion 4 weeks after first IL13-PE infusion.

* Letters $(a, b, c)$ indicate sequence of chemotherapeutic regimens.

erin deposition in the right pons at the site of the previous catheter tip. Increased perfusion in the left side of pons remained unchanged from before infusion. The 4-week postoperative scan showed tumor enlargement, more extensive enhancement, and increased perfusion in enhancing and nonenhancing tumor. The patient died 1 week later.

The 1-week postoperative MRS demonstrated large lactate peaks bilaterally in the pons, higher on the left. The relative amounts of NAA, choline, and creatine were normal on the right side, but NAA was reduced on the left. The 4-week follow-up MRS study continued to demonstrate a very large pontine lactate peak and elevated pontine and midbrain choline levels.

\section{Patient 3}

Hospital Course. The patient was neurologically stable on postoperative day 1. By the next day, he demonstrated improvement in the left-sided weakness that had been present preoperatively. He was discharged on postoperative day 7 without complication. The day following his second CED treatment, the patient had increased left-sided weakness and difficulty with speech and swallowing. His weakness, dysphagia, and speech difficulty improved, but not to pre-infusion levels during his 5-day hospitalization.

Quality of Life. The Adaptive Behavior subscale score decreased from the baseline value of 63.6 to 54.5 at the 4 -week follow-up. The child engaged less often in fun activities and rested more during the day. The scores for Psychological Functioning and Medical/Physical Status changed slightly (respectively, from 79.5 at baseline to 84.1 at 4 weeks post-infusion and from 73.1 at baseline to 67.3 at 4 weeks post-infusion). The CNS Problems score dropped from 91.7 to 79.2 because of problems paying attention and taking longer to complete tasks.

Anatomical MRI and MRS. One week after the first infusion, MRI demonstrated stable tumor size, enhancement, and tumor blood volume relative to preoperative findings (Fig. 2A). The 4-week postoperative scan demonstrated decreased heterogeneous enhancement and stable FLAIR signal (Fig. 2B). The MRI scan performed 5 days after the second infusion demonstrated markedly reduced hyperperfusion in the DIPG, suggesting treatment response, and stable tumor size and enhancement. Eight weeks after the initial treatment (4 weeks after the second treatment), the tumor size was stable, but new foci of enhancement arose in the pons, midbrain, and thalamus (Fig. $2 \mathrm{C})$. The 12-week postoperative scan demonstrated interval DIPG progression based on increased size and more enhancing foci, blood volume in enhancing areas, and vasogenic edema (Fig. 2D). The patient withdrew from the study and died 23 weeks later.

\section{Patient 4}

Hospital Course. The day after infusion the patient developed lethargy, increasing weakness, and mutism. A complex partial seizure occurred the next day and resolved with intravenous lorazepam. The weakness and alertness improved, but the mutism persisted and was still present at discharge 5 days after infusion.

Quality of Life. The patient did not have complete QOL data for either time point.

Anatomical MRI and MRS. The 1-week postoperative scan demonstrated stable tumor size and heterogeneous enhancement. The 4-week postoperative MRI showed decreased central enhancement but new tumor invasion into the left cerebral peduncle and increased tumor perfusion. MRI performed 2 weeks later for clinical purposes demonstrated enlargement of the pontine and left cerebral peduncle enhancement and elevated pontine perfusion. The patient died 3 weeks later.

The MRS study performed 1 week after infusion demonstrated high lactate peaks and elevated choline to creatine ratio. The 4-week post-infusion scan showed further increase in choline and decrease in lactate and NAA within the mass. A postoperative examination done 2 weeks later for clinical reasons demonstrated low creatine and choline peaks and markedly elevated lactate peaks in areas of abnormal enhancement.

\section{Patient 5}

Hospital Course. Dysarthria developed on the day after the first infusion but improved to normal by the following day. The patient was discharged on post-infusion day 3 . The day after the second infusion, increased movement on the left side was noted. Elevated creatine kinase was treated with IV hydration and normalized by discharge on post-infusion day 4.

Quality of Life. The patient's Adaptive Functioning and Psychological Functioning scores decreased slightly 
TABLE 3. Evaluations before and after IL13-PE infusion

\begin{tabular}{|c|c|c|c|c|c|}
\hline Variable \& Time from 1st Infusion & Pt 1 & Pt 2 & Pt 3 & Pt 4 & Pt 5 \\
\hline \multicolumn{6}{|l|}{ KPS/LPS } \\
\hline$-1 w k$ & 90 & 60 & 90 & 70 & 50 \\
\hline $1 \mathrm{wk}$ & 40 & 60 & 70 & 40 & 50 \\
\hline 4 wks & 80 & 50 & 90 & 70 & 50 \\
\hline 8 wks & $x$ & $\mathrm{x}$ & 70 & 60 & 50 \\
\hline 12 & $x$ & $x$ & 50 & $x$ & 50 \\
\hline \multicolumn{6}{|l|}{ DXM requirement (mg) } \\
\hline 4 wks & 3 & 12 & 24 & 3 & 1 \\
\hline 8 wks & $x$ & $x$ & 4 & $\mathrm{x}$ & 0.75 \\
\hline 12 wks & $x$ & $x$ & 18 & $x$ & $x$ \\
\hline \multicolumn{6}{|l|}{ QOL total scores } \\
\hline$-1 w k$ & 77.5 & - & 77.0 & - & 51.4 \\
\hline 4 wks & 64.6 & - & 71.3 & - & 54.7 \\
\hline \multicolumn{6}{|l|}{ MRI } \\
\hline \multicolumn{6}{|l|}{$1 \mathrm{wk}$} \\
\hline Size & Nrml & Nrml & $\mathrm{Nrml}$ & $\mathrm{Nrml}$ & Nrml \\
\hline Enhancement & Nrml & Nrml & $\mathrm{Nrml}$ & $\mathrm{Nrml}$ & Nrml \\
\hline Perfusion & Nrml & $\mathrm{Nrml}$ & ++ & $\mathrm{Nrml}$ & Nrml \\
\hline \multicolumn{6}{|l|}{4 wks } \\
\hline Size & Nrml & ++ & Nrml & ++ & Nrml \\
\hline Enhancement & ++ & ++ & $\mathrm{Nrml}$ & $\downarrow$ & $\mathrm{Nrml}$ \\
\hline Perfusion & Nrml & $\mathrm{Nrml}$ & $\mathrm{Nrml}$ & Nrml & Nrml \\
\hline \multicolumn{6}{|l|}{8 wks } \\
\hline Size & Nrml & $x$ & Nrml & Nrml & Nrml \\
\hline Enhancement & + & $x$ & ++ & ++ & ++ \\
\hline Perfusion & Nrml & $x$ & $\mathrm{Nrml}$ & ++ & + \\
\hline \multicolumn{6}{|l|}{12 wks } \\
\hline Size & ++ & $x$ & ++ & $\mathrm{x}$ & Nrml \\
\hline Enhancement & ++ & $x$ & ++ & $x$ & $\mathrm{Nrml}$ \\
\hline Perfusion & Nrml & $x$ & $\mathrm{Nrml}$ & $x$ & ++ \\
\hline \multicolumn{6}{|l|}{ MRS } \\
\hline \multicolumn{6}{|l|}{-1 wk } \\
\hline NAA & $\downarrow$ & $x$ & $\downarrow$ & $x$ & $\downarrow$ \\
\hline Choline & $\uparrow$ & $x$ & $\uparrow$ & $x$ & $\uparrow$ \\
\hline Lactate & Nrml & $x$ & $\downarrow$ & $x$ & Nrml \\
\hline \multicolumn{6}{|l|}{$1 \mathrm{wk}$} \\
\hline NAA & $\downarrow$ & $\downarrow$ & $x$ & $\downarrow$ & $\downarrow$ \\
\hline Choline & $\uparrow$ & $\uparrow$ & $x$ & $\uparrow$ & $\uparrow$ \\
\hline Lactate & Nrml & $\uparrow$ & $x$ & $\uparrow$ & Nrml \\
\hline \multicolumn{6}{|l|}{4 wks } \\
\hline NAA & $x$ & $\downarrow$ & $x$ & $\downarrow$ & $\downarrow$ \\
\hline Choline & $x$ & $\uparrow$ & $x$ & $\uparrow$ & $\uparrow$ \\
\hline Lactate & $x$ & $\uparrow$ & $x$ & $\downarrow$ & $\uparrow$ \\
\hline \multicolumn{6}{|l|}{8 wks } \\
\hline NAA & $\downarrow$ & $x$ & $\downarrow$ & $\downarrow$ & $\downarrow$ \\
\hline Choline & $\uparrow$ & $x$ & $\uparrow$ & $\uparrow$ & $\uparrow$ \\
\hline Lactate & Nrml & $x$ & Nrml & $\uparrow$ & Nrml \\
\hline
\end{tabular}

» CONTINUED FROM PREVIOUS COLUMN

TABLE 3. Evaluations before and after IL13-PE infusion

\begin{tabular}{lccccc}
\hline Variable \& Time from 1st Infusion & Pt 1 & Pt 2 & Pt 3 & Pt 4 & Pt 5 \\
\hline MRS (continued) & & & & & \\
\hline 12 wks & & & & & \\
\hline NAA & $\downarrow$ & x & x & x & $\downarrow$ \\
\hline Choline & $\uparrow$ & x & x & x & $\uparrow$ \\
\hline Lactate & Nrml & x & x & x & Nrml \\
\hline Survival (wks) after 1st infusion & 22.0 & 4.7 & 35.6 & 9.3 & 24.3 \\
\hline
\end{tabular}

$\mathrm{DXM}=$ dexamethasone; $\mathrm{nrml}=$ normal $($ stable $) ;+=$ slightly greater than normal; $++=$ moderate increase compared to normal; $x=$ no data at timepoint; $\uparrow=$ increase; $\downarrow$ = decrease

between baseline and 4-week follow-up assessment (from 54.5 to 50 and from 61.4 to 54.5 , respectively). His Medical/Physical Status score improved (from 48.1 to 55.8) due to fewer problems sleeping and headaches. His CNS Problems score improved (from 41.7 to 58.3), with fewer memory problems and difficulties with schoolwork, but the parents may have reported fewer schoolwork difficulties 4 weeks post-infusion because the child attended school less frequently.

Anatomical MRI and MRS. The 1-week postoperative MRI study showed stable tumor size with microhemorrhage at the previous cannula tip location compared to preoperative MRI (Fig. 3A). The 4-week postoperative MRI study showed tumor necrosis, suggesting treatment response (Fig. 3B), with no signal enhancement in the tumor. Perfusion was absent in the necrotic part of the tumor and low or normal elsewhere in the tumor. MRI performed 3 days after the second infusion demonstrated increased pontine and medullary tumor enhancement and blood volume. An MRI study obtained 8 weeks after the initial infusion (4 weeks after the second infusion) demonstrated stable tumor size, increased enhancement, and
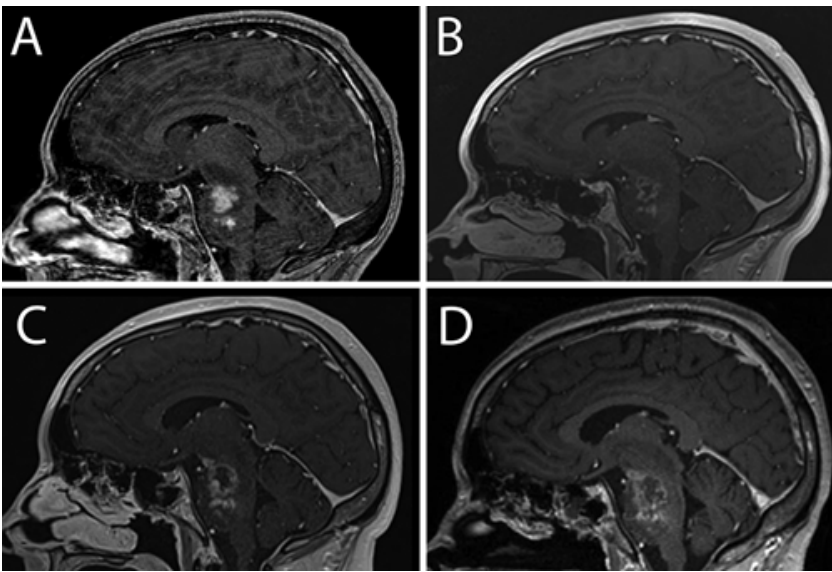

FIG. 2. Patient 3. Sagittal contrast-enhanced T1-weighted MR images obtained preoperatively (A) and 1 month (B) and 2 (C) and 3 (D) months postoperatively. The 1-month follow-up image (B) shows minimal enhancement, low perfusion, and necrosis at the infusion site consistent with treatment response. 

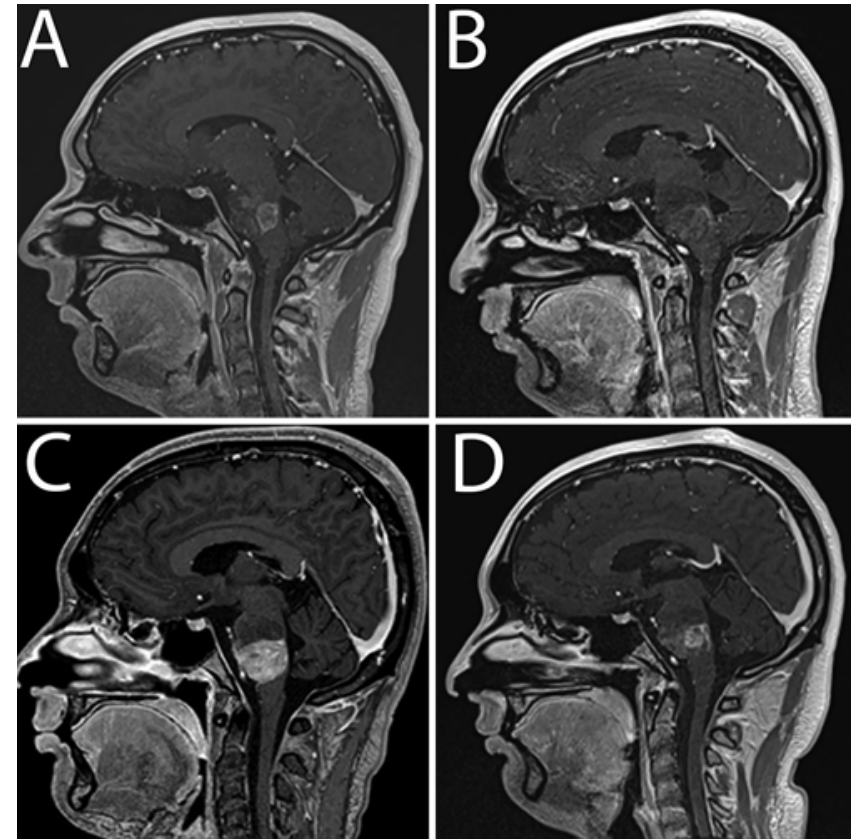

FIG. 3. Patient 5. Sagittal contrast-enhanced T1-weighted MR images obtained preoperatively (A) and 1 month (B) and 2 (C) and 3 (D) months postoperatively. The 1-month follow-up image (B) shows minimal enhancement, low perfusion, and necrosis at the infusion site consistent with treatment response.

areas of hypo- and hyperperfusion (Fig. 3C). The 12-week postoperative scan showed stable tumor size and enhancement, with slightly increased blood volume in enhancing tumor (Fig. 3D). The patient withdrew from the study, entered a chemotherapeutic trial, and died 12 weeks later. The preoperative MRS findings were as follows: NAA/ creatine ratio of 0.60 , choline/creatine ratio of 4.47 , and choline/NAA ratio of 7.44 (Fig. 4A), compared to NAA/ creatine ratio of 10.4 , choline/creatine ratio of 38.3 , and choline/NAA ratio of 3.68 (Fig. 4B) 1 month postoperatively and NAA/creatine ratio of 0.31 , choline/creatine ratio of 2.76 , and choline/NAA ratio of 8.93 (Fig. 4C) 3 months postoperatively.

\section{Discussion}

CED is a regional drug delivery method that uses small hydrostatic pressure gradients to drive bulk flow of drug through the CNS extracellular spaces. Drug distributes uniformly within the treatment volume and little drug enters surrounding tissue. ${ }^{3,4} \mathrm{CED}$ delivered IL13-PE38QQR widely throughout the brain and brainstem in rat and nonhuman primate glioma models., $3,18,31,32$

IL13-PE38QQR, a recombinant chimeric cytotoxin, delivered by CED was selected for clinical trial testing for several reasons. IL13-PE38QQR has been shown to be cytotoxic to glioma cells and nontoxic to normal brain at clinical doses. CED provided higher IL13-PE38QQR levels in tumor tissue than normal brain, and little IL13PE38QQR passed from the brain to the systemic circulation. ${ }^{5}$ In preclinical testing, intratumoral administration of IL13-PE had much greater antitumor efficacy than intravenous or intraperitoneal administration in U251 tumorbearing mice. ${ }^{13}$ Moreover, in clinical studies intratumoral and peritumoral infusion of IL13-PE38QQR was well tolerated. ${ }^{17,19,21}$ However, IL13-PE38QQR did not show survival benefit compared to carmustine wafers in a Phase III randomized trial of the 2 treatments in patients with recurrent glioblastoma. ${ }^{20}$

Brainstem infusion of IL13-PE38QQR by CED was reported in a 4-year-old child with recurrent DIPG. ${ }^{24}$ MRI demonstrated tumor stability at 4 weeks post-treatment but recurrence at 2 months, and the child died 4 months posttreatment. This initial experience prompted this dose-escalation study to further evaluate the safety and possible efficacy against DIPG of IL13-PE38QQR delivered by CED. ${ }^{3}$ In this larger study of 5 children, CED of IL13-PE into the DIPG was technically feasible and produced generally mild and temporary side effects.

Previous studies examining convection-enhanced infusate distribution in naïve nonhuman primate brainstems showed $\mathrm{Vd} / \mathrm{Vi}$ ratios ranging from $6: 1$ to $10: 1$ in this region of compacted fiber tracts. ${ }^{23,29} \mathrm{The} \mathrm{Vd} / \mathrm{Vi}$ ratio in our patients was significantly lower (ranging from 1.6:1 to 4.1:1) than in nonhuman primates, ${ }^{3,24}$ likely because tumor-related vasogenic edema expanded the extracellular space. $^{24,34}$
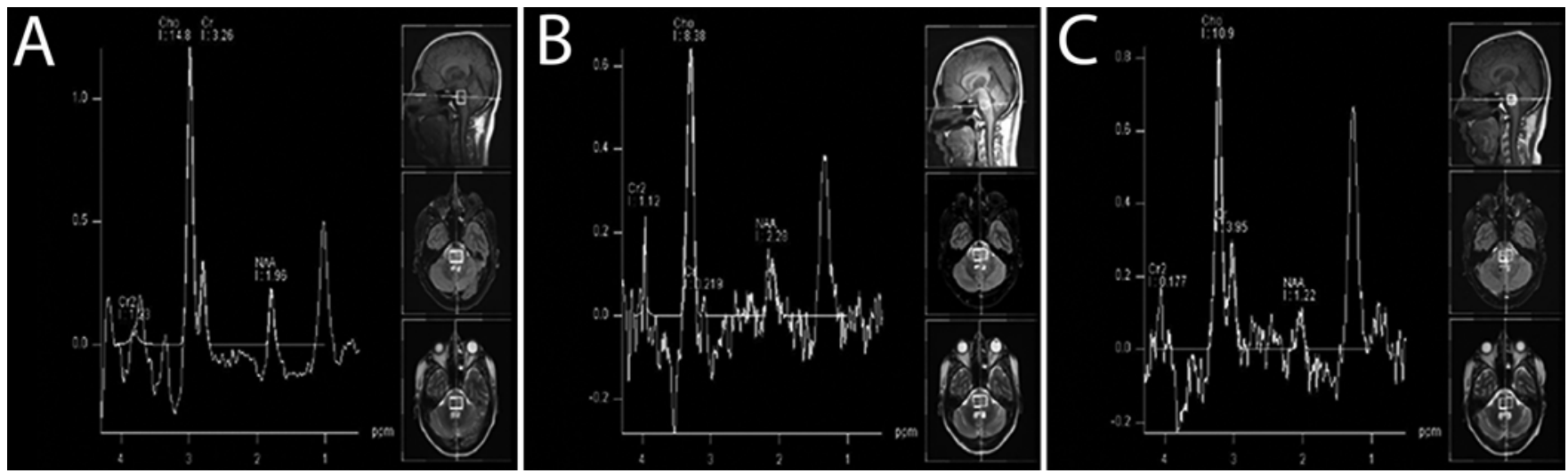

FIG. 4. Patient 5. MRS findings. A: Before initial infusion: NAA/creatine $(\mathrm{Cr})$ ratio of 0.60 , choline $(\mathrm{Cho}) / \mathrm{Cr}$ ratio of 4.47 , and $\mathrm{Cho} /$ NAA ratio of 7.44. B: One month postoperatively: NAA/Cr ratio of 10.4, Cho/Cr ratio of 38.3, and Cho/NAA ratio of 3.68. C: Three months postoperatively: NAA/Cr ratio of 0.31 , Cho/Cr ratio of 2.76 , and Cho/NAA ratio of 8.93 . 

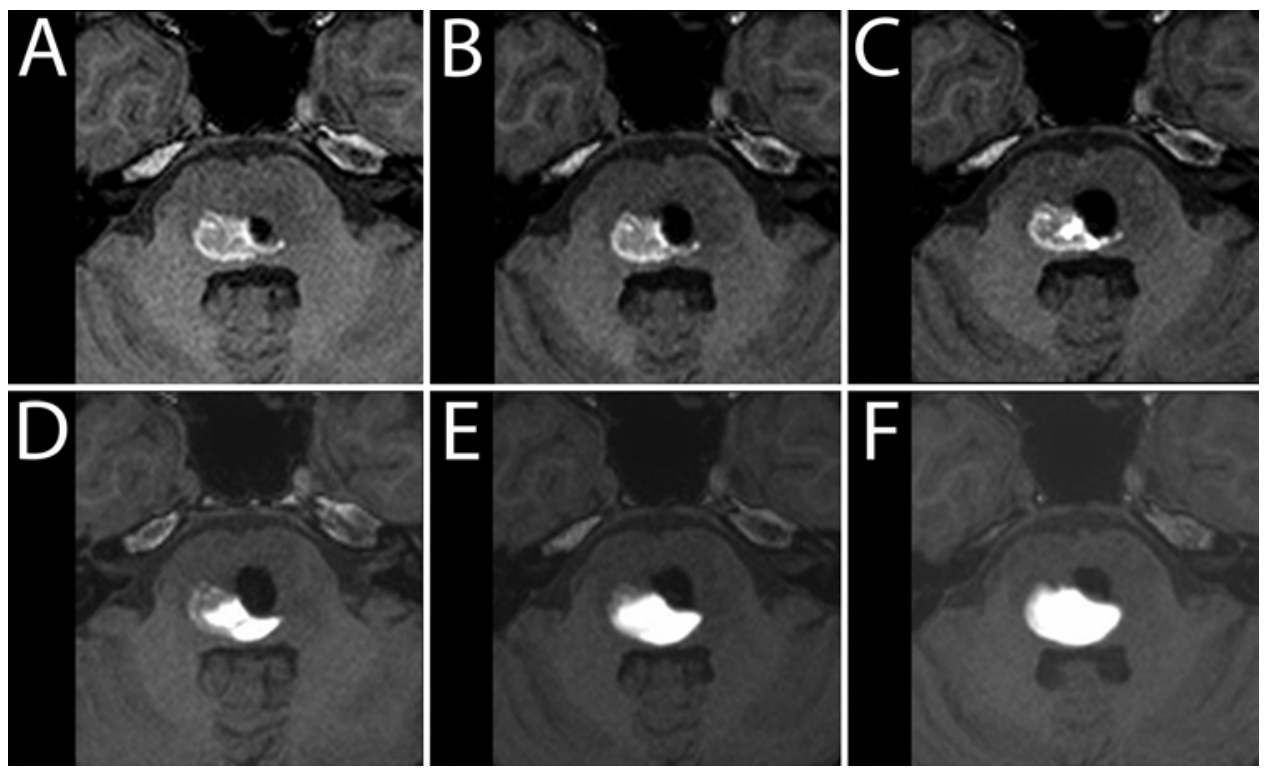

FIG. 5. Patient 2. Intraparenchymal air effects on infusate distribution. A-F: Serial axial T1-weighted MR images obtained every 50 minutes, demonstrating the impact of an intraparenchymal air bubble (hypointensity) introduced at the start of the infusion. While there was a small decrease in the volume of air over time, the serial images demonstrate the restriction of anteromedial spread of infusate (hyperintensity) by the air in the parenchyma.

During patient 5's first infusion, intraoperative MRI showed an air bubble preventing infusate from being distributed by convection throughout the pons (Fig. 5A-F). Imaging during CED procedures detects such drug-distribution problems, which can be corrected by repositioning the infusion cannula. Cannula tracts from previous infusions can critically impact infusate distribution, ${ }^{3}$ as seen during patient 5's second infusion, in which infusate flowed in a retrograde manner into a 4-week-old cannula tract. Advancing the infusion cannula deeper into the tumor and away from the previous tract stopped passage of infusate into the tract.

No patient withdrew from the study because of adverse events or experienced a dose-limiting toxicity. ${ }^{33}$ One persistent grade 3 toxicity occurred: patient 4 did not recover verbalization after the procedure (Table 1). IL13-PE CED otherwise had acceptable toxicity in DIPG patients. KPS/ LPS scores showed a decrease on evaluation 1 week after infusion but returned to baseline or near baseline in 4 of 5 subjects within 4 weeks of infusion (Table 3). QOL scores in 3 of 5 patients generally aligned with functional or radiological data. Decreased QOL in patients 1 and 3 corresponded with decreased KPS/LPS scores. Patient 5 had a treatment response based on MRI findings, clinical data, and improved QOL subscale scores. Parent perception of QOL was consistent with medical indicators.

Conventional MRI alone may inaccurately evaluate DIPG treatment response because imaging changes after treatment are nonspecific, arising from recurrent tumor, inflammatory response, or tumor necrosis, ${ }^{22}$ and poorly correlate with survival. ${ }^{11}$ Disease progression correlates better with H-MRS than perfusion-weighted MRI. ${ }^{31}$ MR spectroscopy can identify characteristic tumor-cell metabolism. Choline, creatine, and NAA are the most common metabolites analyzed. Choline is a tumor tissue biomarker because increased membrane turnover or cellular density elevates choline levels. ${ }^{9}$ Creatine is a biomarker of energy metabolism and levels are usually stable but are decreased in necrotic tissue. ${ }^{916}$ NAA is a biomarker of neuronal integrity. ${ }^{9}$ Increased choline/creatine and choline/NAA ratios identify metabolic changes characteristic of tumor ${ }^{12,22}$ because choline increases, creatine stays stable or decreases, and NAA decreases in tumor tissue. In our study, MRS performed 4 weeks after IL13-PE infusion suggested antitumor effects only in patients 3 and 5 .

The mean survival after the first infusion was 20.3 weeks (Table 2). The trial's limited therapeutic efficacy has several possible explanations. 1) The single catheter could infuse local tumor but not the entire MRI-visible tumor; a multicatheter approach could have distributed infusate to more tumor tissue. 2) Although preclinical studies of IL13-PE38QQR showed antitumor effects against lowgrade and high-grade glioma cell lines, IL13-PE38QQR was not tested against only-recently-available DIPG-derived cell lines. ${ }^{4,10,30} 3$ ) The expression of tumor-specific IL13R in the infused tumors was unknown. In other studies, 33\% to 37\% of DIPGs had normal IL-13 receptor immunohistochemical staining. ${ }^{14,26}$ Even when DIPG had IL13R reactivity, the reactivity was more often moderate ( 8 of 10 cases) than intense ( 2 of 10 cases). ${ }^{26}$ Tumors with intense IL13R expression would be more responsive to the antitumor effect of IL13-PE38QQR.

This study is important because few Phase I clinical trials have assessed the use of CED in treating DIPG. ${ }^{3,28}$ DIPG carries the poorest prognosis of all pediatric brain tumors. Clinical safety, imaging, and outcome methods from this trial could be used in future DIPG clinical trials.

\section{Conclusions}

Direct brainstem infusion of IL13-PE using CED in patients with DIPG temporarily arrested disease progression 
in 2 of 5 patients. However, DIPG had progressed in all 5 patients by 12 weeks after initial infusion. Infusion side effects included exacerbation of preexisting deficits, transient cranial nerve deficits, and lethargy. These side effects reversed with corticosteroid treatment and were related to infusion-related brainstem edema rather than IL13-PE cytotoxicity. No patient had MRI evidence of acute or longterm treatment toxicity.

\section{Acknowledgments}

This research was supported by the Intramural Research Program of the National Institute of Neurological Disorders and Stroke at the National Institutes of Health.

\section{References}

1. Albright AL, Packer RJ, Zimmerman R, Rorke LB, Boyett J, Hammond GD: Magnetic resonance scans should replace biopsies for the diagnosis of diffuse brain stem gliomas: a report from the Children's Cancer Group. Neurosurgery 33:1026-1030, 1993

2. Broniscer A, Gajjar A: Supratentorial high-grade astrocytoma and diffuse brainstem glioma: two challenges for the pediatric oncologist. Oncologist 9:197-206, 2004

3. Chittiboina P, Heiss JD, Warren KE, Lonser RR: Magnetic resonance imaging properties of convective delivery in diffuse intrinsic pontine gliomas. J Neurosurg Pediatr 13:276282, 2014

4. Debinski W, Gibo DM, Hulet SW, Connor JR, Gillespie GY: Receptor for interleukin 13 is a marker and therapeutic target for human high-grade gliomas. Clin Cancer Res 5:985-990, 1999

5. Debinski W, Obiri NI, Pastan I, Puri RK: A novel chimeric protein composed of interleukin 13 and Pseudomonas exotoxin is highly cytotoxic to human carcinoma cells expressing receptors for interleukin 13 and interleukin 4. J Biol Chem 270:16775-16780, 1995

6. Debinski W, Obiri NI, Powers SK, Pastan I, Puri RK: Human glioma cells overexpress receptors for interleukin 13 and are extremely sensitive to a novel chimeric protein composed of interleukin 13 and pseudomonas exotoxin. Clin Cancer Res 1:1253-1258, 1995

7. García-Fiñana M, Cruz-Orive LM, Mackay CE, Pakkenberg $\mathrm{B}$, Roberts N: Comparison of MR imaging against physical sectioning to estimate the volume of human cerebral compartments. Neuroimage 18:505-516, 2003

8. Gholamin S, Mitra SS, Feroze AH, Liu J, Kahn SA, Zhang $\mathrm{M}$, et al: Disrupting the CD47-SIRP $\alpha$ anti-phagocytic axis by a humanized anti-CD47 antibody is an efficacious treatment for malignant pediatric brain tumors. Sci Transl Med 9:eaaf2968, 2017

9. Gujar SK, Maheshwari S, Björkman-Burtscher I, Sundgren PC: Magnetic resonance spectroscopy. J Neuroophthalmol 25:217-226, 2005

10. Halvorson KG, Barton KL, Schroeder K, Misuraca KL, Hoeman C, Chung A, et al: A high-throughput in vitro drug screen in a genetically engineered mouse model of diffuse intrinsic pontine glioma identifies BMS-754807 as a promising therapeutic agent. PLoS One 10:e0118926, 2015

11. Hargrave D, Chuang N, Bouffet E: Conventional MRI cannot predict survival in childhood diffuse intrinsic pontine glioma. J Neurooncol 86:313-319, 2008

12. Howe FA, Barton SJ, Cudlip SA, Stubbs M, Saunders DE, Murphy M, et al: Metabolic profiles of human brain tumors using quantitative in vivo ${ }^{1} \mathrm{H}$ magnetic resonance spectroscopy. Magn Reson Med 49:223-232, 2003

13. Husain SR, Joshi BH, Puri RK: Interleukin-13 receptor as a unique target for anti-glioblastoma therapy. Int J Cancer 92:168-175, 2001

14. Joshi BH, Puri RA, Leland P, Varricchio F, Gupta G, Kocak $M$, et al: Identification of interleukin-13 receptor $\alpha 2$ chain overexpression in situ in high-grade diffusely infiltrative pediatric brainstem glioma. Neuro Oncol 10:265-274, 2008

15. Kawakami M, Kawakami K, Puri RK: Interleukin-4-Pseudomonas exotoxin chimeric fusion protein for malignant glioma therapy. J Neurooncol 65:15-25, 2003

16. Kinoshita Y, Yokota A: Absolute concentrations of metabolites in human brain tumors using in vitro proton magnetic resonance spectroscopy. NMR Biomed 10:2-12, 1997

17. Kioi M, Husain SR, Croteau D, Kunwar S, Puri RK: Convection-enhanced delivery of interleukin-13 receptor-directed cytotoxin for malignant glioma therapy. Technol Cancer Res Treat 5:239-250, 2006

18. Kunwar S: Convection enhanced delivery of IL13-PE38QQR for treatment of recurrent malignant glioma: presentation of interim findings from ongoing phase 1 studies. Acta Neurochir Suppl 88:105-111, 2003

19. Kunwar S, Chang S, Prados M, Berger MS, Sampson JH, Croteau D, et al: Safety of intraparenchymal convectionenhanced delivery of cintredekin besudotox in early-phase studies. Neurosurg Focus 20(4):E15, 2006

20. Kunwar S, Chang S, Westphal M, Vogelbaum M, Sampson J, Barnett G, et al: Phase III randomized trial of CED of IL13-PE38QQR vs Gliadel wafers for recurrent glioblastoma. Neuro Oncol 12:871-881, 2010

21. Kunwar S, Prados MD, Chang SM, Berger MS, Lang FF, Piepmeier JM, et al: Direct intracerebral delivery of cintredekin besudotox (IL13-PE38QQR) in recurrent malignant glioma: a report by the Cintredekin Besudotox Intraparenchymal Study Group. J Clin Oncol 25:837-844, 2007

22. Laprie A, Pirzkall A, Haas-Kogan DA, Cha S, Banerjee A, Le TP, et al: Longitudinal multivoxel MR spectroscopy study of pediatric diffuse brainstem gliomas treated with radiotherapy. Int J Radiat Oncol Biol Phys 62:20-31, 2005

23. Lonser RR, Walbridge S, Garmestani K, Butman JA, Walters HA, Vortmeyer AO, et al: Successful and safe perfusion of the primate brainstem: in vivo magnetic resonance imaging of macromolecular distribution during infusion. J Neurosurg 97:905-913, 2002

24. Lonser RR, Warren KE, Butman JA, Quezado Z, Robison RA, Walbridge $S$, et al: Real-time image-guided direct convective perfusion of intrinsic brainstem lesions. Technical note. J Neurosurg 107:190-197, 2007

25. Office of Human Research Protections: Special protections for children as research subjects. HHS.gov. March 18, 2016 (https://www.hhs.gov/ohrp/regulations-and-policy/guidance/ special-protections-for-children/index.html) [Accessed October 8, 2018]

26. Okada H, Low KL, Kohanbash G, McDonald HA, Hamilton RL, Pollack IF: Expression of glioma-associated antigens in pediatric brain stem and non-brain stem gliomas. J Neurooncol 88:245-250, 2008

27. Pollack IF: Brain tumors in children. N Engl J Med 331:1500-1507, 1994

28. Souweidane MM, Kramer K, Pandit-Taskar N, Zhou Z, Haque S, Zanzonico P, et al: Convection-enhanced delivery for diffuse intrinsic pontine glioma: a single-centre, doseescalation, phase 1 trial. Lancet Oncol 19:1040-1050, 2018

29. Souweidane MM, Occhiogrosso G, Mark EB, Edgar MA: Interstitial infusion of IL13-PE38QQR in the rat brain stem. J Neurooncol 67:287-293, 2004

30. Truffaux N, Philippe C, Paulsson J, Andreiuolo F, GuerriniRousseau L, Cornilleau G, et al: Preclinical evaluation of dasatinib alone and in combination with cabozantinib for the treatment of diffuse intrinsic pontine glioma. Neuro Oncol 17:953-964, 2015 
31. Tzika AA, Astrakas LG, Zarifi MK, Zurakowski D, Poussaint TY, Goumnerova L, et al: Spectroscopic and perfusion magnetic resonance imaging predictors of progression in pediatric brain tumors. Cancer 100:1246-1256, 2004

32. Wolters PL, Martin S, Merker VL, Gardner KL, Hingtgen $\mathrm{CM}$, Tonsgard JH, et al: Patient-reported outcomes in neurofibromatosis and schwannomatosis clinical trials. Neurology 81 (21 Suppl 1):S6-S14, 2013

33. Wolters PL, Martin S, Tamula MA, Wiener L, Perez L, Aikin A, et al: Development of a quality of life scale for children with chronic illness with CNS involvement: preliminary data. Neuro Oncol 6:448-449, 2004 (Abstract)

34. Wood JD, Lonser RR, Gogate N, Morrison PF, Oldfield EH: Convective delivery of macromolecules into the naive and traumatized spinal cords of rats. J Neurosurg 90 (1 Suppl):115-120, 1999

\section{Disclosures}

The authors report no conflict of interest concerning the materi- als or methods used in this study or the findings specified in this paper.

\section{Author Contributions}

Conception and design: Heiss, Warren, Lonser. Acquisition of data: Heiss, Martin, Wolters, Warren, Lonser. Analysis and interpretation of data: all authors. Drafting the article: Heiss, Jamshidi, Shah, Martin, Wolters, Argersinger. Critically revising the article: Heiss, Shah, Argersinger. Reviewed submitted version of manuscript: all authors. Approved the final version of the manuscript on behalf of all authors: Heiss. Statistical analysis: Heiss. Administrative/technical/material support: Argersinger. Study supervision: Heiss, Lonser.

\section{Correspondence}

John D. Heiss: National Institute of Neurological Disorders and Stroke, National Institutes of Health, Bethesda, MD. heissj@ ninds.nih.gov. 